\title{
Misfit elastic energy and a continuum model for epitaxial growth with elasticity on vicinal surfaces
}

\author{
Yang Xiang* \\ Department of Mathematics, Hong Kong University of Science and Technology, Clear Water Bay, Kowloon, Hong Kong
}

Weinan E

Department of Mathematics and PACM, Princeton University, Princeton, New Jersy 08544, USA

(Received 21 March 2003; revised manuscript received 26 August 2003; published 23 January 2004)

\begin{abstract}
In heteroepitaxial growth, the mismatch between the lattice constants in the film and the substrate causes misfit strain in the film, making a flat surface unstable to small perturbations. This morphological instability is called Asaro-Tiller-Grinfeld (ATG) instability. In practice, most devices are fabricated on vicinal surfaces which consist of steps and terraces. In this case, the misfit strain causes step bunching and traditional continuum models for the ATG instability do not apply directly. In this paper, we present a continuum model for this elastically driving step bunching. We validate our model by performing linear stability analysis and numerical simulation in the nonlinear regime. We also present a continuum description for the misfit elastic energy on vicinal surface which incorporates the underlying atomic features of the vicinal surface.
\end{abstract}

DOI: 10.1103/PhysRevB.69.035409 PACS number(s): 81.10.Aj, 81.15.-z, 62.20.Dc, 68.35.-p

\section{INTRODUCTION}

Nanostructures such as quantum wires and quantum dots exhibit novel electronic and optical properties and have important potential applications in semiconductor technology. How to fabricate them efficiently has raised intense interest recently. One promising way is to employ the selforganization process during heteroepitaxial growth of thin films where they are under stress. The mismatch between the lattice constants in the film and in the substrate causes misfit strain and stress in the film, driving the self-organization of surface morphology. Therefore understanding the mechanism of misfit related self-organization is an important step to make this technology into reality.

The stress-driven morphological instability was first studied by Asaro and Tiller ${ }^{1}$ and later independently by Grinfeld $^{2,3}$ and Srolovitz. ${ }^{4}$ It is called Asaro-Tiller-Grinfeld (ATG) instability. These authors studied the linear instability of a planar surface of a stressed solid to small perturbations and found that the planar surface is unstable for perturbations with wave numbers less than a critical value. This instability is manifested by a mass transport via surface diffusion. The stress in the solid is a destabilizing factor while the surface energy is a stabilizing one. This instability was also studied by Spencer et al. ${ }^{5,6}$ Gao, ${ }^{7,8}$ Nozières, ${ }^{9,10}$ Freund and Jonsdottir, ${ }^{11}$ Grilhe, ${ }^{12}$ Yakobson, ${ }^{13}$ and others. Caroli et al. ${ }^{14}$ and Leo and Sekerka ${ }^{15}$ studied the effect of misfit stress on the morphological stability of a growing solid sphere in a supersaturated melt and a growing precipitate in solid solution, respectively. Grinfeld ${ }^{16-18}$ also studied a wide range of different stress driven rearrangement instabilities which include various interfaces, free boundaries, and inclusions. Therefore these instabilities are also referred to as Grinfeld instabilities.

The nonlinear evolution of the stress-driven instability for thick films will result in the formation of cusps. The first fully nonlinear analysis of this instability was performed numerically by Yang and Srolovitz. ${ }^{19,20}$ They showed that this surface instability creates a groove that sharpens as it grows deeper. Chiu and $\mathrm{Gao}^{21}$ were able to solve for the stress field analytically when the surface of the solid is of cycloid type and showed that the near cusps are driven to become more cusplike. Spencer and Meiron ${ }^{22}$ tracked the branch of steadystate solutions numerically and found that the steady-state solution branch terminates as the solutions form a cusp singularity. They also considered the evolution of the instability and found that the formation of cusps was a general feature. Kassner and Misbah ${ }^{23}$ also numerically analyzed the nonlinear evolution of a uniaxially stressed solid. If the films are thin and wet the substrates, Stranski-Krastanow wetting islands will form. The steady states of island shapes were studied by Spencer and Tersoff, ${ }^{24,25}$ Kukta and Freund, ${ }^{26}$ Spencer, ${ }^{27}$ Rudin and Spencer, ${ }^{28}$ Shanahan and Spencer, ${ }^{29}$ and others. The nonlinear evolution of the surfaces of thin films and the formation of islands were studied by Chiu and Gao, ${ }^{30}$ and Zhang and Bower. ${ }^{31}$

These authors treated the surface as a continuum, neglecting the presence of steps. This can be true only at relatively high temperature above the roughening transition, when the surface can change continuously. The normal temperature for epitaxial growth is below the roughening transition, when the surface consists only of steps and terraces (see, e.g., Pimpinelli and Villain ${ }^{32}$ ). In this case, the surface cannot change continuously and it has been shown that there is an activation barrier for the nucleation of steps. ${ }^{33,34}$ Therefore the continuum theories mentioned above do not apply directly. In practice, most semiconductor devices are fabricated on vicinal surfaces. Such surfaces are cut at a small angle to the atomic planes, creating a succession of terraces separated by atomic-height steps. The mismatch between lattice constants in the film and in the substrate also causes misfit strain in the film, but the self-organization is achieved by step bunching. ${ }^{35-38}$ These bunches have uniform size and spacing, and they are much straighter than single steps, which tend to meander ${ }^{39}$ due to step edge barrier (Schwoebel barrier). ${ }^{40}$ Therefore they can serve as superior templates for growth of quantum wires and nucleation of clusters. ${ }^{41-46}$

The understanding of step bunching induced by misfit 
strain is not as complete as that of the traditional ATG instability for a continuous surface. One important model was proposed by Tersoff et al. ${ }^{47,48}$ describing the dynamics of each step based on Burton-Cabrera-Frank (BCF) theory. ${ }^{49}$ In their model, the elastic effect of a step on a thick substrate is modeled by a force monopole caused by misfit stress in the bulk and a force dipole caused by the step. ${ }^{50}$ The force monopole causes attractive interaction between successive steps, which destabilizes a uniform step train. The force dipole causes repulsive interaction between successive steps, which stabilizes a uniform step train. They analyzed the linear instability toward step bunching from a uniform step train to small perturbations and showed that it evolves by progressive coalescence of step bunches. ${ }^{47}$ They also studied the kinetic debunching effect and demonstrated numerically how to control the size of bunches. ${ }^{48}$ Another important model was proposed by Duport et al. ${ }^{51-53}$ Besides the attractive and repulsive elastic interactions between steps, they also considered the elastic interaction between adatoms and steps and the Schwoebel barrier, ${ }^{40}$ both of which depend on the deposition flux. The elastic interaction between adatoms and steps is stabilizing or destabilizing depending on the sign of the misfit. The Schwoebel barrier, which the adatoms on the terraces must overcome in order to get incorporated into the downward steps, is always stabilizing. They also gave the dependence of the step-step interactions on the misfit, the force dipole of a step, and the elastic constants. Kaganer and Ploog ${ }^{54,55}$ studied the island shapes and the growth kinetics of step bunches. They also showed that the interaction between the strain field of a step may give rise to a negative line energy of steps. ${ }^{55}$ Duport et al. ${ }^{56}$ computed the equilibrium pyramidlike island shapes. All these models are twodimensional, based on the observation that the steps are very straight in bunches. Kukta and Bhattacharya ${ }^{57}$ proposed a three-dimensional model for step-flow-mediated crystal growth under stress.

In most cases, a continuum model is desired when we are interested only in the shapes of step bunches or islands. The existing continuum models for the traditional ATG instability follow Mullins' chemical-potential argument. ${ }^{58}$ They do not incorporate the atomic structure of the underlying crystal which plays an important role in epitaxial growth. Holy et al. ${ }^{59}$ introduced a continuum description of this problem based on the work by Tersoff et al. ${ }^{47}$ using step density as the variable. Shenoy and Freund ${ }^{60}$ proposed a continuum description of the energetics and evolution of stepped surfaces in strained nanostructures and applied it to study the evolution of surface shape of an epitaxial film being deposited onto a substrate.

In this paper, we present a continuum model for step bunching induced by elasticity. We obtain our continuum model by taking the continuum limit from the discrete models of Tersoff et al. ${ }^{47}$ and Duport et al. ${ }^{53}$ therefore it incorporates the underlying atomic features. We validate our model by performing linear stability analysis and numerical simulation in the nonlinear regime. We also present a continuum description of the misfit elastic energy on the vicinal surface. It contains an additional term which represents the underlying step-terrace structure of the vicinal surface com- pared with the traditional misfit elastic energy of a slightly perturbed surface above roughening transition. ${ }^{1-6,11}$ Without lattice misfit, it is well known that the surface energy of the vicinal surface is different from that of the continuous surface above roughening transition. ${ }^{32}$ However, as far as we know, the difference between the continuum descriptions of the misfit elastic energy on the vicinal surface and that on the continuous surface above roughening transition has not been studied in the literature.

The main difference between our continuum model and the model of Shenoy and Freund ${ }^{60}$ is that their model emphasizes the growth mode of island formation where there is no energy barrier to the nucleation of islands due to the dependence of the formation energy of steps on the misfit strain, which is another effect of the misfit and the underlying atomic structure; on the other hand, our model is focused on the step bunching instability in the step flow growth mode without nucleation, featuring the new misfit elastic energy on vicinal surface mentioned above. Besides, flux dependent effects such as step edge barriers and the elastic interaction between adatoms and steps are also included in our model. Compared with the continuum description proposed by Holy et al. ${ }^{59}$ unlike the simple average used by them, our model is derived rigorously by taking the continuum limit from the discrete models; ${ }^{47,53}$ no artificial cutoff as in Holy et al. ${ }^{59}$ is needed for the elastic interactions between steps.

The rest of this paper is organized as follows. In Sec. II, we review the concept of epitaxial growth on vicinal surface and the BCF model, and present the discrete models of Duport et al. ${ }^{53}$ and Tersoff et al. ${ }^{47}$ In Sec. III, we present our continuum model and the continuum description for the misfit elastic energy on vicinal surface. In Sec. IV, we study the linear instability of a planar surface to small perturbations and compare our results with those of the discrete models and models for the traditional ATG instability. In Sec. V, we study numerically the nonlinear evolution of small perturbations and compare our results with those of the discrete models. In Sec. VI, we study numerically the equilibrium states of step bunches and explain their coalescence. In Sec. VII, we present our conclusion.

\section{EPITAXIAL GROWTH ON VICINAL SURFACE AND BCF-LIKE MODELS}

In this section, we review the concept of epitaxial growth on vicinal surface and the BCF theory, and present the discrete models for elastically driving step bunching.

Epitaxial growth is the growth of crystalline film on a crystalline substrate following the same structure as the substrate. According to the BCF theory, ${ }^{49}$ the surface of the film is divided by steps into terraces. The height of a step is one lattice constant. The adatoms diffuse on the terraces until they meet the steps and get incorporated into the steps. The surface then grows (see Fig. 1). Vicinal surface consists of successive steps and the angle between the surface and the crystallographic plane is small. The best way to grow a good crystal is to grow it on a vicinal surface through a regular flow of steps. ${ }^{32}$

Steps and adatoms can interact elastically. The elastic in- 


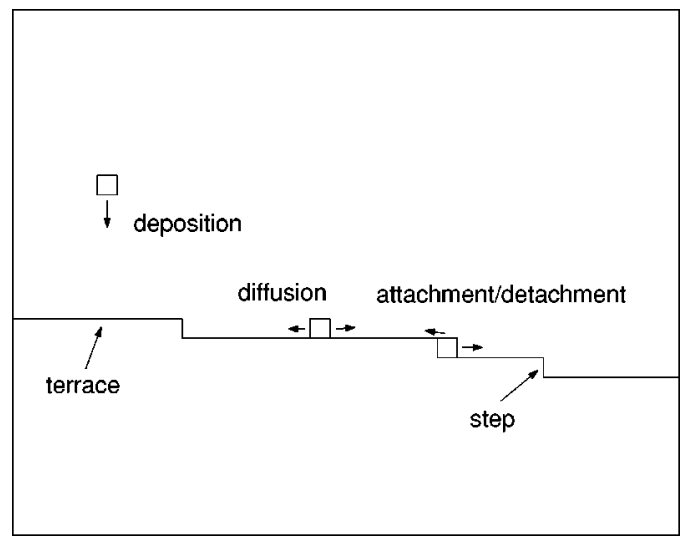

FIG. 1. Schematic picture for the BCF theory.

teraction may be due to different kinds of mechanisms. One mechanism is the broken bond mechanism, which originates from the force dipole exerted by adatoms or steps due to the broken bonds of the adatoms or at the steps. The other mechanism is the misfit mechanism, which originates from the misfit between the lattice constants of the film and the substrate. The misfit strain and stress exist in the bulk of the film. If the modulation of the surface is small, the effect of this mechanism is equivalent to a surface stress acting on a flat surface proportional to the modulation of the surface and the misfit. In the case of isotropic misfit and Hooke's law, the surface stress due to misfit is given by

$$
\eta_{x x}=\eta_{y y}=\frac{\delta a}{a} \frac{E}{1-\nu} \delta h,
$$

where $a$ is the lattice constant in the substrate, $a+\delta a$ is the lattice constant in the film, $E$ is the Young modulus, $\nu$ is the Poisson ratio, and $\delta h$ is the modulation of the surface.

The elastic interactions between adatoms and steps affect the diffusion of the adatoms on terraces. The elastic interactions between steps modify the equilibrium adatom density on steps and therefore modify the incorporating process of adatoms into the steps if we assume that the adatoms incorporated into the steps can detach from them.

As far as diffusion alone is concerned, the adatoms prefer to hop to the upper step rather than to the lower step, since an energy barrier exists near the lower step, this is called the Schwoebel barrier. ${ }^{40}$ Let $k^{+}$and $k^{-}$be the hopping rates of adatoms to the upper steps and lower steps, respectively. Then the Schwoebel effect stipulates

$$
k^{+} \geqslant k^{-} \text {. }
$$

Now we present the model given by Duport et al. ${ }^{53}$ for $1+1$ dimensional vicinal surface based on the BCF theory. The $1+1$ dimensional model assumes that the steps are straight and parallel, and the diffusion on the terrace is uniform in the direction parallel to the steps. Without loss of generality, it is assumed that the terrace to the left of a step is higher than that to the right. Letting $\left\{x_{n}\right\}$ with $\cdots<x_{n-1}$ $<x_{n}<x_{n+1}<\cdots$ be the step train, the equations describing the adatom diffusion and step motion can be written as

$$
\begin{cases}\frac{\partial \rho_{n}}{\partial t}=D \frac{\partial}{\partial x}\left(\frac{\partial \rho_{n}}{\partial x}+\frac{\rho_{n}}{k_{B} T} \frac{\partial U}{\partial x}\right)+F, & x_{n}<x<x_{n+1}, \\ D\left(\frac{\partial \rho_{n}}{\partial x}+\frac{\rho_{n}}{k_{B} T} \frac{\partial U}{\partial x}\right)=k^{+}\left(\rho_{n}-\rho_{n}^{0}\right), & x=x_{n} \\ D\left(\frac{\partial \rho_{n}}{\partial x}+\frac{\rho_{n}}{k_{B} T} \frac{\partial U}{\partial x}\right)=-k^{-}\left(\rho_{n}-\rho_{n+1}^{0}\right), & x=x_{n+1} \\ \frac{d x_{n}(t)}{d t}=a^{2}\left[k^{+}\left(\left.\rho_{n}\right|_{x=x_{n}^{+}}-\rho_{n}^{0}\right)+k^{-}\left(\left.\rho_{n-1}\right|_{x=x_{n}^{-}}-\rho_{n}^{0}\right)\right],\end{cases}
$$

where $\rho_{n}$ is the adatom density on the terrace between the $n$th step and the $(n+1)$ th step, $F$ is the deposition flux, $D$ is the diffusion constant on terraces, $k_{B}$ is the Boltzmann constant, $T$ is the temperature, $\rho_{n}^{0}$ is the equilibrium adatom density on the $n$th step, it is equal to the equilibrium adatom density on a step in the absence of elastic interactions $\rho_{0}$ with a local correction due to elasticity,

$$
\rho_{n}^{0}=\rho_{0} e^{-\left(1 / k_{B} T\right)\left[U\left(x_{n}\right)-f_{n}\right]} .
$$

The function $U(x)$ is the elastic energy due to the interaction between an adatom and the steps

$$
U(x)=-\sum_{m=-\infty}^{+\infty} \frac{\alpha_{0}}{x_{m}-x}
$$

and $f_{n}$ describes the elastic interactions between the $n$th step and all the other steps

$$
f_{n}=-\sum_{m \neq n}\left(\frac{\alpha_{1}}{x_{m}-x_{n}}-\frac{\alpha_{2}}{\left(x_{m}-x_{n}\right)^{3}}\right) .
$$

The constants $\alpha_{1}, \alpha_{2}>0, \alpha_{0}$ may be either positive or negative, and

$$
\begin{aligned}
& \alpha_{0}=\frac{2}{\pi}(1+\nu) \frac{\delta a}{a} \mu_{a} a^{3}, \\
& \alpha_{1}=\frac{2 E}{\pi} \frac{1+\nu}{1-\nu}\left(\frac{\delta a}{a}\right)^{2} a^{4}, \\
& \alpha_{2}=\frac{4}{\pi E}\left(1-\nu^{2}\right) \mu_{s}^{2} a^{4},
\end{aligned}
$$

where $a^{2} \mu_{a}$ is the force dipole moment due to the broken bond mechanism of an adatom, $a \mu_{s}$ is the force dipole moment density due to the broken bond mechanism along a step.

The first equation in the system (3) describes the deposition and diffusion processes of adatoms on a terrace. The second and third equations describe the incorporating process of adatoms into the steps. The fourth equation gives the velocity of the steps.

The function $U(x)$ describes the elastic interaction between one adatom and the steps. In this expression, the adatom has the broken bond effect and the steps have the misfit effect, which is dominant among all possible combinations. The sign of $\alpha_{0}$ can be either positive or negative depending 
on the sign of $\mu_{a} \delta a$. The case of $\mu_{a} \delta a>0$ means that the elastic interaction between an adatom with the broken bond effect and a step with the misfit effect is repulsive for upper steps, and is attractive for lower steps. Thus the adatoms on a terrace prefer to hop to the lower step than to the upper one. On the other hand, $\mu_{a} \delta a<0$ means that the elastic interaction between an adatom with the broken bond effect and a step with the misfit effect is attractive for upper steps, and is repulsive for lower steps. Thus the adatoms on a terrace prefer to hop to the upper step than to the lower one.

The function $f_{n}$ describes the elastic interactions between steps. The first term in it comes from the elastic interaction between steps due to the misfit mechanism. It is an attractive interaction. The second term comes from the elastic interaction between steps due to the broken bond mechanism. It is a repulsive interaction. Here we do not consider the interactions between a step with the broken bond effect and another step with the misfit effect, since they cancel. In fact, consider two successive steps, one is higher than the other. Due to the misfit mechanism, the higher step generates a surface stress with a sign the same as $\delta a$ and the lower step generates a surface stress with a sign the same as $-\delta a$. However, due to the broken bond mechanism, the two steps generate force dipole moments with the same sign. Therefore the elastic interaction between one step with the broken bond mechanism and another step with the misfit mechanism cancel and we do not need to take it into consideration. Duport et al. ${ }^{53}$ did not notice this and they omitted this interaction by assuming that either of the two mechanisms is dominant.

Usually in epitaxial growth, the deposition process is much slower than the diffusion process, which means that the step velocity is very small compared with the adatom hopping velocity. Therefore the quasistatic approximation can be made,

$$
\frac{\partial \rho_{n}}{\partial t} \approx 0
$$

Under this assumption, the system (3) has an explicit solution. If it is further assumed that the elastic energies are very small compared to the thermal energy

$$
f_{n}, U(x) \ll k_{B} T,
$$

keeping the leading-order terms of $1 / k_{B} T$ for each effect, we can write the step velocity as

$$
\begin{aligned}
\frac{1}{a^{2}} \frac{d x_{n}(t)}{d t}= & F \frac{l_{n}+l_{n-1}}{2}+\frac{\rho_{0}}{k_{B} T} \frac{l_{n}}{\frac{1}{k^{+}}+\frac{1}{k^{-}}+\frac{l_{n}}{D}} \frac{f_{n+1}-f_{n}}{l_{n}} \\
& -\frac{\rho_{0}}{k_{B} T} \frac{l_{n-1}}{\frac{1}{k^{+}}+\frac{1}{k^{-}}+\frac{l_{n-1}}{D} \frac{f_{n}-f_{n-1}}{l_{n-1}}} \\
& \frac{F l_{n}}{2}\left(\frac{1}{k^{-}}-\frac{1}{k^{+}}\right) \\
+ & \frac{1}{k^{+}}+\frac{1}{k^{-}}+\frac{l_{n}}{D}-\frac{1}{\left.\frac{1}{k^{+}}+\frac{1}{k^{-}}+\frac{1}{k^{-}}-\frac{1}{k^{+}}\right)}
\end{aligned}
$$

$$
\begin{aligned}
& +\frac{\frac{F}{D} \int_{x_{n}}^{x_{n+1}}\left(y-\frac{x_{n+1}+x_{n}}{2}\right) \frac{U(y)}{k_{B} T} d y}{\frac{1}{k^{+}}+\frac{1}{k^{-}}+\frac{l_{n}}{D}} \\
& -\frac{\frac{F}{D} \int_{x_{n-1}}^{x_{n}}\left(y-\frac{x_{n}+x_{n-1}}{2}\right) \frac{U(y)}{k_{B} T} d y}{\frac{1}{k^{+}}+\frac{1}{k^{-}}+\frac{l_{n-1}}{D}},
\end{aligned}
$$

where

$$
l_{n} \equiv x_{n+1}-x_{n}
$$

is the width of the terrace between the $n$th step and the ( $n$ $+1)$ th step.

This step motion equation is simpler than that derived by Duport et al. ${ }^{53}$ We keep only the leading-order terms of $1 / k_{B} T$ for each effect, respectively, while they kept all $O\left(1 / k_{B} T\right)$ terms.

If we consider only the attractive and repulsive elastic interactions between steps and neglect the elastic interaction between adatoms and steps, as well as the Schwoebel barrier, as was done by Tersoff et al., ${ }^{47,48}$ we have

$$
\frac{1}{a^{2}} \frac{d x_{n}}{d t}=F \frac{l_{n}+l_{n+1}}{2}+\frac{\rho_{0} D}{k_{B} T}\left(\frac{f_{n+1}-f_{n}}{l_{n}}-\frac{f_{n}-f_{n-1}}{l_{n-1}}\right) .
$$

This corresponds to the case $U(y) \equiv 0$ and $k^{+}=k^{-}=+\infty$ in (12).

\section{CONTINUUM EQUATION AND THE MISFIT ELASTIC ENERGY FOR VICINAL SURFACE}

In this section, we present our continuum model governing the step bunching induced by elasticity. It is obtained by taking continuum limit from the modified discrete model of Duport et al. Eq. (12), of which the discrete model (14) of Tersoff et al. is a special case. We have assumed that the lattice constant $a$ is very small compared with the length scale in which we are interested, then the surface can be considered as a continuous function $h(x)$. The details of the derivation can be found in the paper by Xiang. ${ }^{61}$

Our continuum equation is

$$
\begin{aligned}
h_{t}= & a^{3} F\left[1+\frac{a^{2}}{12} \frac{\partial^{2}}{\partial x^{2}}\left(\frac{1}{h_{x}^{2}}\right)\right] \\
& +\frac{a^{2} \pi \alpha_{1} \rho_{0} D}{k_{B} T} \frac{\partial}{\partial x}\left(\frac{1}{1-(L / a) h_{x}} \frac{\partial f}{\partial x}\right) \\
& +\frac{1}{2} a^{3} F\left(l_{-}-l_{+}\right) \frac{\partial}{\partial x}\left(\frac{1}{1-(L / a) h_{x}}\right) \\
& +\frac{a^{3} F \alpha_{0}}{k_{B} T} \frac{\partial}{\partial x}\left(\frac{\ln \left(2 \pi\left|h_{x}\right|\right)}{1-(L / a) h_{x}}\right),
\end{aligned}
$$


where

$$
f(x)=-\frac{1}{\pi} \int_{-\infty}^{+\infty} \frac{h_{x}(y)}{x-y} d y+\frac{a}{2 \pi} \frac{h_{x x}}{h_{x}}+\frac{\pi l_{e}^{2}}{2 a} h_{x} h_{x x}
$$

and parameters

$$
\begin{gathered}
l_{e}=\sqrt{\frac{\alpha_{2}}{\alpha_{1}},} \\
l_{ \pm}=\frac{D}{k^{ \pm}}, \\
L=l_{+}+l_{-} .
\end{gathered}
$$

This equation applies to the step flow growth mode without nucleation on vicinal surface. The surface is assumed to be monotonic and without loss of generality, we assume $h_{x}$ $<0$.

The function $f(x)$ represents the elastic interactions between steps. It is the continuum limit of Eq. (6) [the factor $\pi \alpha_{1}$ has been moved to the coefficient in the continuum equation (15)]. The first two terms in $f$ come from the misfit interaction between steps $[1 / r$ summation in Eq. (6)]. The third term comes from the broken bond interaction between steps $\left[1 / r^{3}\right.$ summation in Eq. (6)]. The logarithm term in the continuum equation (15) comes from the elastic interaction between adatoms and steps, which is given by Eq. (5) in the discrete model. The parameter $l_{e}$ represents the equilibrium distance between two successive steps under the attractive misfit interaction and the repulsive broken bond interaction, the parameters $l_{+}$and $l_{-}$represent the strength of the upward and downward step edge barriers, respectively, and

$$
l_{-} \geqslant l_{+}
$$

due to the Schwoebel effect.

The first term in $f(x)$ is the Hilbert transform of $h_{x}$ with a negative sign. The Hilbert transform of a function $u(x)$ is defined by

$$
H(u)=\frac{1}{\pi} \int_{-\infty}^{+\infty} \frac{u(y)}{x-y} d y .
$$

We can rewrite the continuum equation as

$$
h_{t}=a^{3} F-\frac{\partial}{\partial x} J(x, t),
$$

where the surface flux $J(x, t)$ is

$$
\begin{aligned}
J(x, t)= & -\frac{a^{5}}{12} F \frac{\partial}{\partial x}\left(\frac{1}{h_{x}^{2}}\right)-\frac{a^{2} \pi \alpha_{1} \rho_{0} D}{k_{B} T} \frac{1}{1-(L / a) h_{x}} \frac{\partial f}{\partial x} \\
& -\frac{1}{2} a^{3} F\left(l_{-}-l+\right) \frac{1}{1-(L / a) h_{x}} \\
& -\frac{a^{3} F \alpha_{0}}{k_{B} T} \frac{\ln \left(2 \pi\left|h_{x}\right|\right)}{1-(L / a) h_{x}} .
\end{aligned}
$$

The term $a^{3} F$ in the equation is the average growth rate of the surface. The first term in $J(x, t)$ represents the asymmetric effect due to the local surface profile. The third term in $J(x, t)$ represents the well-known Schwoebel effect. The last term in $J(x, t)$ is the contribution of the adatom-step interaction. Its sign can be positive or negative depending on the sign of $\alpha_{0}$. Recall that $\alpha_{0}>0$ means that the elastic interaction between an adatom and a step is repulsive for upper steps, and is attractive for lower steps. Thus the adatoms on a terrace prefer to hop to the lower step than to the upper one. On the other hand, $\alpha_{0}<0$ means that the elastic interaction between an adatom and a step is attractive for upper steps, and is repulsive for lower steps. Thus the adatoms on a terrace prefer to hop to the upper step than to the lower one. All the three effects mentioned above depend on the deposition flux.

The second term in $J(x, t)$ represents elastic interactions between steps. It is an energetic effect not depending on the deposition flux. The summation of the Hilbert transform term and the $h_{x x} / h_{x}$ term in the expression of $f(16)$ is the chemical potential for surface diffusion due to the misfit interaction between steps. This kind of interaction has a global effect. The $h_{x} h_{x x}$ term in the expression of $f$ is the chemical potential for surface diffusion due to the broken bond interaction between steps, $h_{x x}$ is the curvature (if $h_{x}$ is small), and the coefficient $h_{x}$ reflects the local density of steps. This kind of interaction has only local effect.

If we consider only the elastic interactions between steps and neglect the elastic interaction between adatoms and steps as well as the step edge barriers, the continuum equation (15) becomes

$$
\begin{aligned}
h_{t}= & a^{3} F+\frac{a^{5} F}{12} \frac{\partial^{2}}{\partial x^{2}}\left(\frac{1}{h_{x}^{2}}\right)+\frac{a^{2} \pi \alpha_{1} \rho_{0} D}{k_{B} T} \frac{\partial^{2}}{\partial x^{2}}\left[-H\left(h_{x}\right)\right. \\
& \left.+\frac{a}{2 \pi} \frac{h_{x x}}{h_{x}}+\frac{\pi l_{e}^{2}}{2 a} h_{x} h_{x x}\right] .
\end{aligned}
$$

It corresponds to the case $\alpha_{0}=0$ and $l_{+}=l_{-}=0$ in Eq. (15). It is the continuum limit of the discrete model (14) of Tersoff et al.

If we further neglect the deposition flux, it becomes

$$
\begin{aligned}
h_{t}= & \frac{a^{6} \rho_{0} D}{k_{B} T} \frac{\partial^{2}}{\partial x^{2}}\left[2 E \frac{1+\nu}{1-\nu}\left(\frac{\delta a}{a}\right)^{2}\left(-H\left(h_{x}\right)+\frac{a}{2 \pi} \frac{h_{x x}}{h_{x}}\right)\right. \\
& \left.+\frac{2 \pi\left(1-\nu^{2}\right) \mu_{s}^{2}}{a E} h_{x} h_{x x}\right] .
\end{aligned}
$$

It has a variational form

$$
h_{t}=D_{0} \mu_{x x},
$$

where

$$
D_{0}=\frac{a^{6} \rho_{0} D}{k_{B} T}
$$

is a diffusion constant and 


$$
\begin{aligned}
\mu= & 2 E \frac{1+\nu}{1-\nu}\left(\frac{\delta a}{a}\right)^{2}\left(-H\left(h_{x}\right)+\frac{a}{2 \pi} \frac{h_{x x}}{h_{x}}\right) \\
& +\frac{2 \pi\left(1-\nu^{2}\right) \mu_{s}^{2}}{a E} h_{x} h_{x x}
\end{aligned}
$$

is the chemical potential, which is the variation of the total elastic energy

$$
\begin{aligned}
\mathcal{E}= & \int\left[2 E \frac{1+\nu}{1-\nu}\left(\frac{\delta a}{a}\right)^{2}\left(-\frac{1}{2} \tilde{h} H\left(h_{x}\right)+\frac{a}{2 \pi}\left|h_{x}\right| \ln \left|h_{x}\right|\right)\right. \\
& \left.+\frac{\pi\left(1-\nu^{2}\right) \mu_{s}^{2}}{3 a E}\left|h_{x}\right|^{3}\right] d x .
\end{aligned}
$$

Here $\widetilde{h}(x)$ is the deviation from the flat surface with a slope equal to the average slope of $h(x)$. The first terms in $\mu$ and $\mathcal{E}$ come from the misfit elastic interaction between steps. The second terms in $\mu$ and $\mathcal{E}$ come from the broken bond elastic interaction between steps.

Our continuum equation applies to the elastically driving instability on vicinal surface which consists of steps and terraces and the angle between the surface and the crystallographic plane is small. It is different from the continuum equation for the traditional ATG instability. In the traditional ATG instability, the surface is assumed to be a continuum without the step-terrace structure, which happens at relatively high temperatures above the roughening transition. The evolution of the surface is driven by the misfit elastic energy and surface free energy. The evolution equation is given by ${ }^{1,4-6,11}$

$$
h_{t}=D_{1}\left(1+|\nabla h|^{2}\right)^{1 / 2} \nabla_{S}^{2}(S+\gamma \kappa) \text {, }
$$

where

$$
D_{1}=\frac{D_{S} \delta \Omega^{2}}{k_{B} T}
$$

is the diffusion constant, $S$ is the misfit elastic energy density on the surface, $\kappa$ is the curvature of the surface, $\gamma$ is the surface free energy density of the film, $D_{S}$ is the surface diffusivity of atoms, $\delta$ is the surface density of atoms, $\Omega$ is the atomic volume, $\nabla_{S}$ is the surface gradient. If the modulation of the surface is small, the evolution equation can be written as

$$
h_{t}=D_{1} \frac{\partial^{2}}{\partial x^{2}}\left[-2 E \frac{1+\nu}{1-\nu}\left(\frac{\delta a}{a}\right)^{2} H\left(h_{x}\right)-\gamma h_{x x}\right] \text {. }
$$

In the absence of the misfit elastic energy, it is well known that the surface energies are different for the continuum surface above roughening transition temperature and the vicinal surface consisting of steps and terraces. ${ }^{32}$ For the continuum surface above roughening transition temperature, the surface energy is proportional to the length of the surface (in the two-dimensional case), and its chemical potential is proportional to the curvature of the surface which is the last term in Eq. (32). For the vicinal surface, the surface energy density is

$$
\beta_{0}+\beta_{1}\left|h_{x}\right|+\beta_{2}\left|h_{x}\right|^{3}
$$

and its chemical potential for a monotonic surface with negative slope is

$$
\mu^{\text {surf }}=6 \beta_{3} h_{x} h_{x x} .
$$

It corresponds to the case of $\delta a=0$ in our continuum equation (25). Obviously, our continuum equation recovers the well-known result for the vicinal surface without misfit elastic energy.

Now we focus on the misfit elastic energy. The misfit elastic energy density on the surface serves as the chemical potential for the evolution of the surface. For the continuum surface above roughening transition temperature, the misfit elastic energy density on the surface is the first term in Eq. (32) if the modulation of the surface is small. For the vicinal surface, according to our continuum Eq. (25), the elastic energy density, or the chemical potential due to the misfit elasticity, is given by

$$
\mu^{\text {misfit }}=2 E \frac{1+\nu}{1-\nu}\left(\frac{\delta a}{a}\right)^{2}\left(-H\left(h_{x}\right)+\frac{a}{2 \pi} \frac{h_{x x}}{h_{x}}\right) .
$$

In this continuum description, the leading-order effect of the misfit elastic interaction between steps is the Hilbert transform term, which agrees with the misfit elastic energy density for the traditional ATG instability. The second term is the correction to the misfit elastic energy density due to the discrete nature of the stepped surface. It can be understood as follows. We have shown that to the leading-order approximation,

$$
\sum_{m \neq n} \frac{a}{x_{m}-x_{n}} \approx-\int_{-\infty}^{+\infty} \frac{h_{x} d x}{x-x_{n}}=-\pi H\left(h_{x}\right) .
$$

Since the local step density in the continuum description is $-h_{x} / a$, the integral in the above equation can be understood as the weighted average of the $1 / r$ interaction. However, this simple average is not accurate for a vicinal surface with stepterrace structure. For a given step $x_{n}$ on the vicinal surface, there is no step on its two adjacent terraces $\left(x_{n-1}, x_{n}\right)$ and $\left(x_{n}, x_{n+1}\right)$. Therefore the misfit interaction in a small neighborhood of $x_{n}$ should be subtracted from the simple-average continuum expression. This gives the second term in Eq. (35). The rigorous derivation can be found in the paper by Xiang. ${ }^{61}$ Though this effect is small compared with the leading-order effect of the misfit elastic interaction, it is comparable with the broken bond interaction between steps and becomes important when wide terraces appear, which is the case of step bunching. Physically, perturbations with wavelength less than the terrace width are impossible on the stepped surface without nucleation of new steps. Linear stability analysis in the later section shows that this term stabi- 
lizes the perturbations with wavelength less than the terrace width in a uniform step train, therefore gives the correct physics. The conclusion is still true locally in the nonlinear regime if we notice that the local terrace width is $a /\left|h_{x}\right|$. With this term, we shall show in the later sections that our continuum equation successfully predicts step bunching and the simulation results agree with those of the discrete models.

As our continuum equation, the continuum description of the misfit elastic interaction on vicinal surface (35) applies only to the monotonic surface. It is singular on the top or the valley of the surface where the slope equals zero. Recall that small slope means wide terrace. In fact, a terrace cannot be arbitrarily wide because if it is wide enough, islands will be nucleated on it. Using a simple interpolation, the misfit elastic interaction on vicinal surface can be written as

$$
\mu^{\text {misfit }}=2 E \frac{1+\nu}{1-\nu}\left(\frac{\delta a}{a}\right)^{2}\left(-H\left(h_{x}\right)-\frac{a}{2 \pi} \frac{h_{x x}}{\sqrt{h_{x}^{2}+a^{2} / l_{n c}^{2}}}\right),
$$

where $l_{n c}$ is the maximal terrace width without nucleation. Without nucleation, $l_{n c}=+\infty$, Eq. (35) is recovered (note the assumption of $h_{x}<0$ for it). When nucleation is included, $l_{n c}$ is finite and no terrace wider than $l_{n c}$ exists. Near the top or the valley, from the continuum description, the surface is flat and the slope is 0 ; from the microscopic point of view, the width of the terrace there is approximately $l_{n c}$. Therefore the effect of the second term in Eq. (35) near the top or the valley should be $-l_{n c} h_{x x} / 2 \pi$ based on the discussion in the preceding paragraph. Equation (36) gives exactly this value when $h_{x}=0$. We leave the more careful validation of Eq. (36) for the future work.

\section{LINEAR INSTABILITY}

In this section, we use our continuum equation to study the linear instability for a planar surface to small perturbations and compare our results with those of the discrete models and models for the traditional ATG instability.

\section{A. Linear instability for planar surface}

Our continuum equation (15) has the following solutions representing planar surfaces (uniform step trains):

$$
h_{0}=a^{3} F t-\frac{a}{l} x+c,
$$

where $l$ is the terrace width in the uniform step train, and $c$ is a constant.

Now consider the planar surface with a small perturbation. Let

$$
h=h_{0}+\hat{h},
$$

where
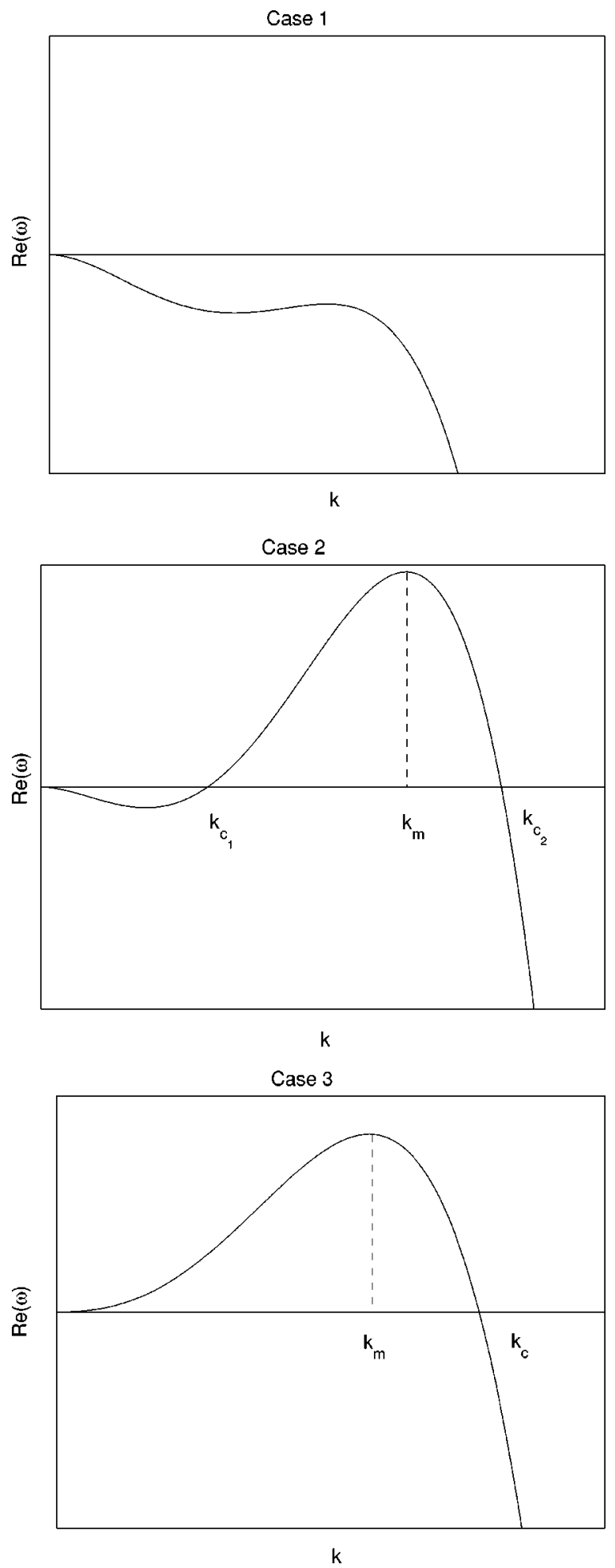

FIG. 2. Three types of linear instabilities.

$$
\hat{h}=\epsilon e^{i k x+\omega t}
$$

with small $\epsilon$. Here without loss of generality, we assume that the wave number $k>0$.

Inserting this expression of $h$ into the continuum equation (15) and keeping the $O(\epsilon)$ terms, we get 


$$
\begin{aligned}
\operatorname{Re}(\omega)= & -\frac{a^{2} \alpha_{1} \rho_{0} D}{k_{B} T} \frac{l^{2}}{2(l+L)}\left(1+\frac{\pi^{2} l_{e}^{2}}{l^{2}}\right) k^{4} \\
& +\frac{a^{2} \pi \alpha_{1} \rho_{0} D}{k_{B} T} \frac{l}{l+L} k^{3}-a^{2} F \frac{l^{2}}{(l+L)^{2}}\left[\frac{1}{2}\left(l_{-}^{2}-l_{+}^{2}\right)\right. \\
& \left.-\frac{\alpha_{0}}{k_{B} T}\left(l+L+L \ln \frac{l}{2 \pi a}\right)\right] k^{2}
\end{aligned}
$$

and

$$
\operatorname{Im}(\omega)=-\frac{1}{6} a^{2} F l^{3} k^{3}
$$

If $\operatorname{Re}(\omega)<0$, the small perturbation decays and the planar surface is stable. On the contrary, if $\operatorname{Re}(\omega)>0$, the small perturbation grows and the planar surface is unstable.

The effect of the broken bond interaction between steps is proportional to $k^{4}$, and it is a stabilizing factor. It stabilizes for large wave numbers. The main effect of the misfit interaction between steps is proportional to $k^{3}$, and it destabilizes for small wave numbers. The misfit interaction between steps also has a small stabilizing effect proportional to $k^{4}$.

The first part in the $k^{2}$ term is due to the Schwoebel effect, it stabilizes for small wave numbers. The second part in the $k^{2}$ term is due to the elastic interaction between adatoms and steps; it can be either stabilizing or destabilizing depending on the sign of $\alpha_{0}$. It also takes its effect for small wave numbers. When $\alpha_{0}>0$, i.e., the adatoms prefer to hop to the lower step, this interaction is destabilizing. When $\alpha_{0}$
$<0$, i.e., the adatoms prefer to hop to the upper step, this interaction is stabilizing. If the net effect of Schwoebel barrier and the elastic interaction between adatoms and steps is to make the upper step preferred by the adatoms, the $k^{2}$ term stabilizes. Otherwise if the net effect makes the lower step preferred by the adatoms, the $k^{2}$ term destabilizes.

The effect of deposition flux is neither stabilizing nor destabilizing. It shifts the fluctuation of the surface.

We discuss three different cases, see Fig. 2.

Case 1. Strong deposition flux $F$ and the upper step is preferred by the adatoms. More precisely,

$$
\begin{aligned}
F & >\frac{\pi^{2} \alpha_{1} \rho_{0} D(l+L)}{2 k_{B} T\left(l^{2}+\pi^{2} l_{e}^{2}\right)\left[\frac{1}{2}\left(l_{-}^{2}-l_{+}^{2}\right)-\frac{\alpha_{0}}{k_{B} T}\left(l+L+L \ln \frac{l}{2 \pi a}\right)\right]} \\
& >0 .
\end{aligned}
$$

In this case, the planar surface is always stable.

Case 2. Weak deposition flux $F$ and the upper step is preferred by the adatoms. More precisely,

$$
F<\frac{\pi^{2} \alpha_{1} \rho_{0} D(l+L)}{2 k_{B} T\left(l^{2}+\pi^{2} l_{e}^{2}\right)\left[\frac{1}{2}\left(l_{-}^{2}-l_{+}^{2}\right)-\frac{\alpha_{0}}{k_{B} T}\left(l+L+L \ln \frac{l}{2 \pi a}\right)\right]} .
$$

In this case, we have two critical wave numbers $k_{c_{1}}$ and $k_{c_{2}}$. The planar surface is unstable if the perturbation wave number $k$ satisfies $k_{c_{1}}<k<k_{c_{2}}$, and is stable otherwise, where

$$
k_{c_{1,2}}=\frac{\pi l\left(1 \mp \sqrt{1-\frac{2 F k_{B} T\left(l^{2}+\pi^{2} l_{e}^{2}\right)}{\pi^{2} \alpha_{1} \rho_{0} D(l+L)}\left[\frac{1}{2}\left(l_{-}^{2}-l_{+}^{2}\right)-\frac{\alpha_{0}}{k_{B} T}\left(l+L+L \ln \frac{l}{2 \pi a}\right)\right]}\right.}{l^{2}+\pi^{2} l_{e}^{2}}
$$

and the most unstable wave number is

$$
k_{m}=\frac{3 \pi l\left(1+\sqrt{1-\frac{16 F k_{B} T\left(l^{2}+\pi^{2} l_{e}^{2}\right)}{9 \pi^{2} \alpha_{1} \rho_{0} D(l+L)}\left[\frac{1}{2}\left(l_{-}^{2}-l_{+}^{2}\right)-\frac{\alpha_{0}}{k_{B} T}\left(l+L+L \ln \frac{l}{2 \pi a}\right)\right]}\right)}{4\left(l^{2}+\pi^{2} l_{e}^{2}\right)}
$$

Case 3. The lower step is preferred by the adatoms. More precisely,

$$
\frac{1}{2}\left(l_{-}^{2}-l_{+}^{2}\right)<\frac{\alpha_{0}}{k_{B} T}\left(l+L+L \ln \frac{l}{2 \pi a}\right) .
$$

In this case, we have one critical wave number $k_{c}$. The planar surface is unstable if the wave number $k$ satisfies $k<k_{c}$ and is stable if $k>k_{c}$, where

$$
k_{c}=\frac{\pi l\left(1+\sqrt{1+\frac{2 F k_{B} T\left(l^{2}+\pi^{2} l_{e}^{2}\right)}{\pi^{2} \alpha_{1} \rho_{0} D(l+L)}\left[\frac{\alpha_{0}}{k_{B} T}\left(l+L+L \ln \frac{l}{2 \pi a}\right)-\frac{1}{2}\left(l_{-}^{2}-l_{+}^{2}\right)\right]}\right.}{l^{2}+\pi^{2} l_{e}^{2}}
$$

and the most unstable wave number is 


$$
k_{m}=\frac{3 \pi l\left(1+\sqrt{1+\frac{16 F k_{B} T\left(l^{2}+\pi^{2} l_{e}^{2}\right)}{9 \pi^{2} \alpha_{1} \rho_{0} D(l+L)}\left[\frac{\alpha_{0}}{k_{B} T}\left(l+L+L \ln \frac{l}{2 \pi a}\right)-\frac{1}{2}\left(l_{-}^{2}-l_{+}^{2}\right)\right]}\right)}{4\left(l^{2}+\pi^{2} l_{e}^{2}\right)} .
$$

\section{B. Comparison with the linear instability result of Duport et al.}

Using their discrete model, Duport et al. ${ }^{53}$ found that the stability condition for a step train with uniform terrace width $l$ is

$$
\begin{aligned}
R(K)= & R_{0}-R_{1} \sum_{m=1}^{\infty} \gamma_{m} \cos (K m) \\
& +R_{2} \sin \frac{K}{2} \sum_{m=1}^{\infty} C_{m} \sin \left[K\left(m-\frac{1}{2}\right)\right] \\
& -R_{3} \sin \frac{K}{2} \sum_{m=1}^{\infty} D_{m} \sin \left[K\left(m-\frac{1}{2}\right)\right]<0,
\end{aligned}
$$

where the small perturbation of the position of the $n$th step is $\zeta(t) \cos [K n-\phi(t)]$, and

$$
\begin{gathered}
R_{0}=-\frac{F\left(l_{-}^{2}-l_{+}^{2}\right)}{2(l+L)^{2}}+\frac{F \alpha_{0}}{k_{B} T}\left(\frac{\ln 2 \pi-\gamma}{l+L}+\frac{L \ln \frac{l}{2 \pi a}}{(l+L)^{2}}\right) \\
+\frac{F \alpha_{0}\left[l L^{2}+2(L-l) l_{+} l_{-}\right]}{a k_{B} T(l+L)^{3}}, \\
R_{1}=\frac{2 F \alpha_{0}}{k_{B} T(l+L)}, \\
R_{2}=\frac{4 \alpha_{1} \rho_{0} D}{k_{B} T(l+L) l^{2}}+\frac{2 F \alpha_{0}\left(l L+4 l_{+} l_{-}\right)}{k_{B} T l(l+L)^{2}}, \\
R_{3}=\frac{12 \alpha_{1} \rho_{0} D l_{e}^{2}}{k_{B} T(l+L) l^{4}}, \\
C_{m}=\sum_{p=m}^{\infty} \frac{1}{p^{2}}, \\
\gamma_{m}=\sum_{p=m}^{\infty}\left(\frac{1}{2 p}+\frac{1}{2(p+1)}-\ln \frac{p+1}{p}\right), \\
\sum_{p=m}^{\infty} \frac{1}{p^{4}}, \\
D_{m}
\end{gathered}
$$

where $\gamma$ is the Euler constant.

The growth rate of this small perturbation is

$$
\frac{d \zeta}{d t}=4 a^{2} \sin ^{2} \frac{K}{2} R(K) \zeta
$$

We consider only the regime when the lattice constant $a$ is very small compared with the length scale in which we are interested. To compare their result with ours, we let $K=k l$ where $k$ is the wave number in the length scale we are interested in. As was done to get our continuum equation, we keep only the leading terms of $a$ and $1 / k_{B} T$ for each kind of effect, ${ }^{63}$ respectively, then their stability function becomes

$$
\begin{aligned}
R(K)= & -\frac{\alpha_{1} \rho_{0} D}{2 k_{B} T} \frac{1}{l+L}\left(1+\frac{\pi^{2} l_{e}^{2}}{l^{2}}\right) k^{2}+\frac{\pi \alpha_{1} \rho_{0} D}{k_{B} T} \frac{1}{(l+L) l} k \\
& -F \frac{1}{(l+L)^{2}}\left[\frac{1}{2}\left(l_{-}^{2}-l_{+}^{2}\right)-\frac{\alpha_{0}}{k_{B} T}\left(l+L+L \ln \frac{l}{2 \pi a}\right)\right] .
\end{aligned}
$$

Therefore, in this regime, the growth rate becomes

$$
\frac{d \zeta}{d t}=a^{2} l^{2} k^{2} R(K) \zeta
$$

It is the same as our dispersion relation (40).

\section{The case of strong flux}

Duport et al. ${ }^{52}$ discussed the case when the elastic interactions between steps are negligible with respect to the Schwoebel effect and the elastic interaction between adatoms and steps. This happens when the flux is relatively strong but still weak enough to make the quasistatic approximation valid.

In this case, our continuum equation becomes

$$
\begin{aligned}
h_{t}= & a^{3} F+\frac{1}{2} a^{3} F\left(l_{-}-l_{+}\right) \frac{\partial}{\partial x}\left(\frac{1}{1-(L / a) h_{x}}\right) \\
& +\frac{a^{3} F \alpha_{0}}{k_{B} T} \frac{\partial}{\partial x}\left(\frac{\ln \left(2 \pi\left|h_{x}\right|\right)}{1-(L / a) h_{x}}\right)
\end{aligned}
$$

and our dispersion relation becomes

$$
\omega=-a^{2} F \frac{l^{2}}{(l+L)^{2}}\left[\frac{1}{2}\left(l_{-}^{2}-l_{+}^{2}\right)-\frac{\alpha_{0}}{k_{B} T}\left(l+L+L \ln \frac{l}{2 \pi a}\right)\right] k^{2} .
$$

If $\alpha_{0}<0$, which means the adatoms prefer to hop to the upper step due to the elastic interaction, $\omega$ is less than 0 , which means the planar surface is always stable. 
If $\alpha_{0}>0$, the adatoms prefer to hop to the lower step due to the elastic interaction. There exists a critical terrace width $l_{c}$ satisfying

$$
\frac{1}{2}\left(l_{-}^{2}-l_{+}^{2}\right)=\frac{\alpha_{0}}{k_{B} T}\left(l_{c}+L+L \ln \frac{l_{c}}{2 \pi a}\right) .
$$

If the terrace width $l<l_{c}$, the net effect of Schwoebel barrier and the elastic interaction between adatoms and steps makes the upper step preferred. In this case, the planar surface is stable. On the other hand if the terrace width $l>l_{c}$, the net effect of Schwoebel barrier and the elastic interaction between adatoms and steps makes the lower step preferred. In this case, the planar surface is unstable.

In conclusion, in the limit of strong flux, whether a planar surface is stable or not depends on the net effect of the Schwoebel barrier and the elastic interaction between adatoms and steps. If the net effect makes the upper step preferred, the planar surface is stable. If this effect makes the lower step preferred, the planar surface is unstable.

If the terrace width is very large, $l \gg l_{+}, l_{-}$, the planar surface will be unstable if

$$
\frac{\alpha_{0} l}{k_{B} T}>\frac{1}{2}\left(l_{-}^{2}-l_{+}^{2}\right) .
$$

These are the assumption and the conclusion in Duport et $a .^{52}$ In this case, only the elastic interaction between adatoms and the two nearest steps is important.

\section{The case of weak flux without Schwoebel barrier}

Tersoff et al. ${ }^{47}$ studied the step train subject to $1 / r$ attractive and $1 / r^{3}$ repulsive interactions between steps. Their step motion equation is Eq. (14). It is a special case of the model of Duport et al. ${ }^{53}$ where there is no Schwoebel effect and the flux is weak so that the elastic interaction between adatoms and steps is negligible.

Considering a step train with uniform terrace width $l$ subject to a small periodic perturbation with $N$ steps in one period: $x_{m}(t)=l(m+F t)+\epsilon e^{r t} \cos [(2 \pi / N) m$ $+(2 \pi / N) F t]$, Tersoff et al. ${ }^{47}$ obtained the dispersion relation

$$
r=\frac{a^{2} \alpha_{1} \rho_{0} D}{k_{B} T} \frac{8 \pi^{4}}{l^{3} N^{3}}\left[1-\frac{1}{N}+\frac{\pi^{2} l_{e}^{2}}{l^{2} N}\left(-1+\frac{2}{N}-\frac{1}{N^{2}}\right)\right] .
$$

On the other hand, in this regime, our continuum equation is Eq. (24) and our dispersion relation is

$$
\operatorname{Re}(\omega)=\frac{a^{2} \pi \alpha_{1} \rho_{0} D}{k_{B} T}\left[k^{3}-\frac{l}{2 \pi}\left(1+\frac{\pi^{2} l_{e}^{2}}{l^{2}}\right) k^{4}\right] .
$$

Recalling that $k$ is the number of periods in $2 \pi$ and $N$ is the number of steps in one period, we have the relation $k N l=2 \pi$. Using this relation, we have

$$
\operatorname{Re}(\omega)=\frac{a^{2} \alpha_{1} \rho_{0} D}{k_{B} T} \frac{8 \pi^{4}}{N^{3} l^{3}}\left[1-\left(1+\frac{\pi^{2} l_{e}^{2}}{l^{2}}\right) \frac{1}{N}\right] .
$$
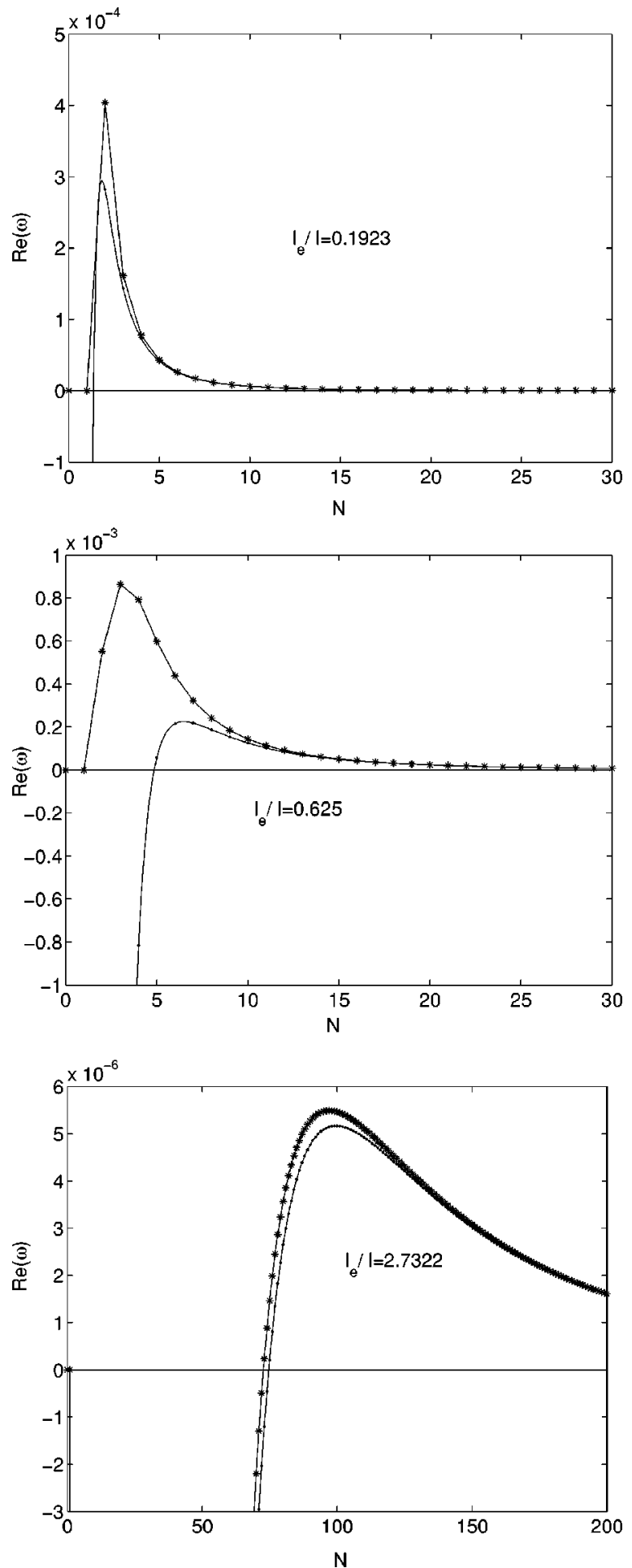

FIG. 3. Comparison of dispersion relations of our continuum model and those of the discrete model of Tersoff et al. The solid line is our result and the line with stars is theirs. The stars are plotted at the integer $N$, which represents the number of steps in one period. We have omitted the factor of $8 \pi^{4} a^{2} \alpha_{1} \rho_{0} D /\left(N^{3} l^{3} k_{B} T\right)$.

Comparing our result (66) with theirs (64), we can see that we have neglected the $O\left(1 / N^{5}\right)$ and $O\left(1 / N^{6}\right)$ terms. The comparison is shown in Fig. $3 .^{64}$ In the first example $l_{e} / l$ $=0.1923 \ll 1$. In the second example $l_{e} / l=0.625$. In the 
third example $l_{e} / l=2.7322 \gg 1$. Recall that $l_{e}=\sqrt{\alpha_{2} / \alpha_{1}}$ is the equilibrium distance between two steps under the two elastic interactions.

From the comparison, we conclude that both models exhibit long wave instability. The predicted instability matches very well in the limit $l_{e} / l \gg 1$, i.e., the equilibrium distance between two adjacent steps under the repulsive and attractive elastic interactions is much longer than the distance between two adjacent steps in the initial uniform step train. In the limit $l_{e} / l \ll 1$, the two predictions are qualitatively similar, but may have different critical wavelengths and most unstable modes.

Now if we consider only the misfit elastic interaction, i.e., $l_{e}=0$, using the perturbation wavelength $\lambda=N l$, the dispersion relation (66) becomes

$$
\operatorname{Re}(\omega)=\frac{a^{2} \alpha_{1} \rho_{0} D}{k_{B} T} \frac{8 \pi^{4}}{N^{3} l^{3}}\left(1-\frac{l}{\lambda}\right) .
$$

The first term comes from the Hilbert transform term in the misfit elastic energy density Eq. (35). The second term comes from the nonlinear term in Eq. (35). We can see that the new nonlinear term in Eq. (35) stabilizes the perturbations with wavelength less than the average terrace width. It gives the correct physics for the stepped surface because the perturbations with wavelength less than the terrace width are impossible without nucleation of new steps.

\section{E. Comparison with the linear instability result of the traditional ATG instability}

In this subsection, we compare our linear instability result with that of the traditional ATG instability. ${ }^{1-6,11}$ The step bunching problem corresponds to a three-dimensional stressed solid with perturbations of its surface only in $x$ direction.

In the traditional ATG instability, the surface is assumed to be a continuum without step-terrace structure, which happens at relatively high temperatures above the roughening transition. The evolution of the surface of a stressed solid is driven by elastic energy and surface free energy. The evolution equation is given by Eq. (30).

For a planar surface of an epitaxially strained solid, the evolution of a linear perturbation $\hat{h}=\epsilon e^{i k x+\omega t}$ has the following dispersion relation

$$
\omega=\frac{D_{S} \delta \Omega^{2}}{k_{B} T}\left[\left(\frac{\delta a}{a}\right)^{2} \frac{2 E(1+\nu)}{1-\nu} k^{3}-\gamma k^{4}\right],
$$

where $a$ is the lattice constant in the substrate and $a+\delta a$ is the lattice constant in the film, $E$ is Young modulus, $\nu$ is Poisson ratio. Here it is assumed that the elastic constants are the same in the film and the substrate.

For the vicinal surface, the corresponding problem is a special case of our continuum model without deposition flux and step edge barriers. Using our continuum equation, the dispersion relation of the linear perturbation for a planar surface representing a uniform step train is

$$
\omega=\frac{D \rho_{0} a^{6}}{k_{B} T}\left(\frac{\delta a}{a}\right)^{2} \frac{2 E(1+\nu)}{1-\nu}\left[k^{3}-\frac{l}{2 \pi}\left(1+\frac{\pi^{2} l_{e}^{2}}{l^{2}}\right) k^{4}\right] .
$$

In the limit when the repulsive interaction is very strong, i.e., $l_{e} \gg l$, our dispersion relation becomes

$$
\omega=\frac{D \rho_{0} a^{6}}{k_{B} T}\left[\left(\frac{\delta a}{a}\right)^{2} \frac{2 E(1+\nu)}{1-\nu} k^{3}-\frac{2 \pi\left(1-\nu^{2}\right) \mu_{s}^{2}}{l E} k^{4}\right] .
$$

We get the same linear instability result as that of the traditional ATG instability. But in the traditional ATG instability, the stabilizing factor is the surface free energy while in our result, the stabilizing factor is the broken bond elastic interaction between steps.

\section{NONLINEAR EVOLUTION}

In this section, we report our results of numerical simulations for the nonlinear evolution of small perturbations to a planar surface. For simplicity, we only consider the misfit and broken bond elastic interactions between steps, neglecting other effects as well as deposition flux. We compare our results with those of the discrete model of Tersoff et al. ${ }^{47}$ which considered the same effects.

Assume that there are $N$ steps in one period. After rescaling $x$ in $N l / 2 \pi, h$ in $N a / 2 \pi$, and time in $l^{3} N^{3} k_{B} T / 8 \pi^{4} a^{2} \alpha_{1} \rho_{0} D$, our continuum equation in this case can be written as

$$
h_{t}=\frac{\partial^{2}}{\partial x^{2}}\left[-H\left(h_{x}\right)+\frac{1}{N}\left(\frac{1}{h_{x}}+r h_{x}\right) h_{x x}\right],
$$

where $H\left(h_{x}\right)$ is the Hilbert transform of $h_{x}$, and

$$
r=\frac{\pi^{2} l_{e}^{2}}{l^{2}} .
$$

Now we have two independent parameters $N$ and $r$. Recall that $N$ is the number of steps in one period, $l$ is the terrace width in the initial uniform step train, and $l_{e}$ is the equilibrium distance between two steps under attractive misfit interaction and repulsive broken bond interaction. In this scale, the initial uniform step train is $h_{x}=-1$.

We solve this equation using the pseudospectral method under periodic boundary condition. We choose a uniform step train with a small perturbation as the initial state. We also simulate the same problem using the discrete model of Tersoff et al. ${ }^{47}$ [Eq. (14) without deposition flux $F$ ] and compare the results obtained by these two models. More details of the numerical methods we use can be found in Xiang's thesis. $^{62}$

In the first example we choose the parameters $l_{e} / l$ $=10 a / 52 a=0.192 \ll 1$, which was used by Tersoff et al. ${ }^{47}$ We simulate a system with 20 steps $(N=20)$. From linear instability theory, the most unstable modes are $k=11$ for our continuum model and $k=10$ for the discrete model, respectively; they are not exactly the same. The results are shown 


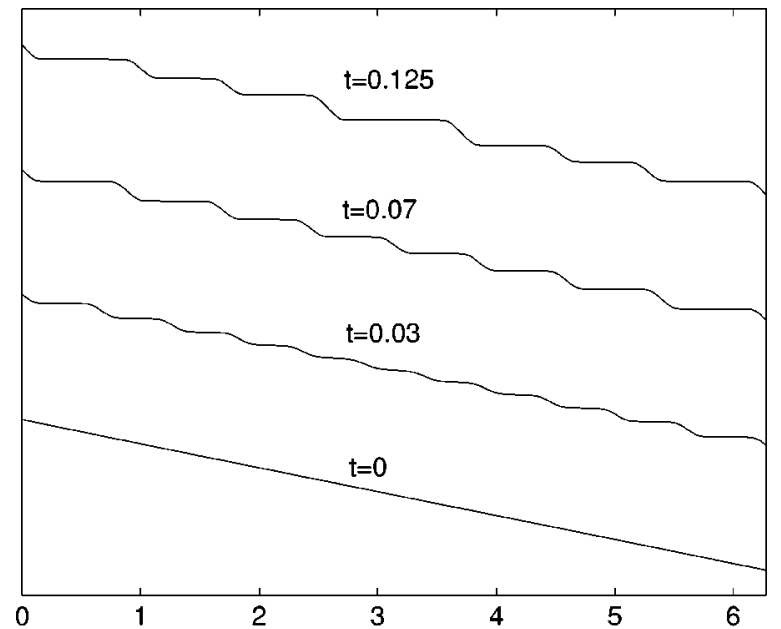

(a)

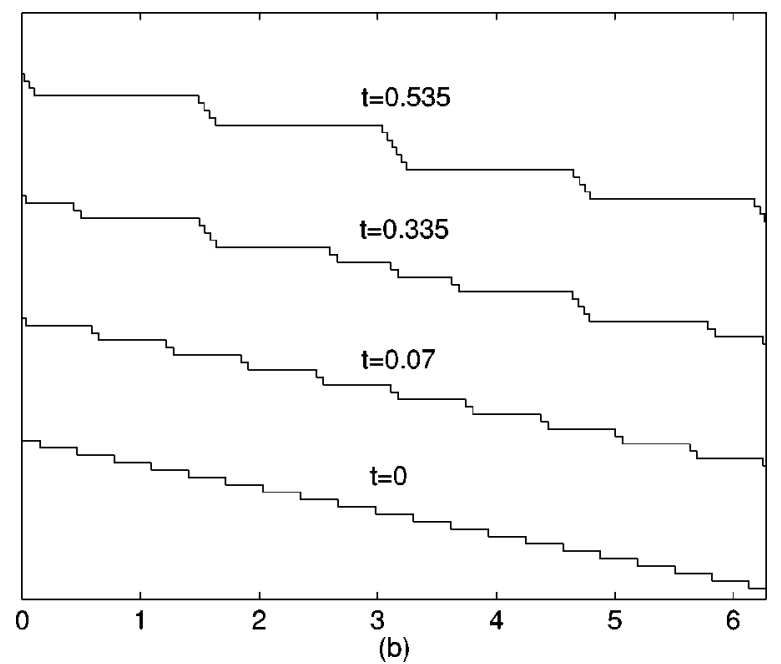

FIG. 4. Evolution of the surface when $l_{e} / l=0.192$. (a) Result of our continuum model. (b) Result of the discrete model.

in Fig. 4. Figure 4(a) is the result of our continuum model and Fig. 4(b) is the result of the discrete model. As predicted by linear instability, the most unstable mode is $k=11$ in our continuum model and $k=10$ in the discrete model. Qualitatively, the result of our continuum model shows step bunching and coalescence of step bunches as the discrete model. The numbers of step bunches at time $t=0.03,0.07$, and 0.125 are 11,8 , and 7 , respectively, in the result of our continuum model, while in the result of the discrete model, the numbers of step bunches at time $t=0.07,0.335$, and 0.535 are 10,8 and 4 , respectively. Figure 5 shows the comparison at time $t=0.07$. The numbers of step bunches in our continuum model and the discrete model are 8 and 10, respectively.

In the second example we choose $l_{e} / l=142 a / 52 a$ $=2.732 \gg 1$. We compute a system with 200 steps $(N$ $=200)$. The most unstable modes for our continuum model and the discrete model are both $k=2$ from linear instability theory. The results are shown in Fig. 6. In this regime, the results of our continuum model and the discrete model agree quantitatively.

From the comparison, we can see that the results of our

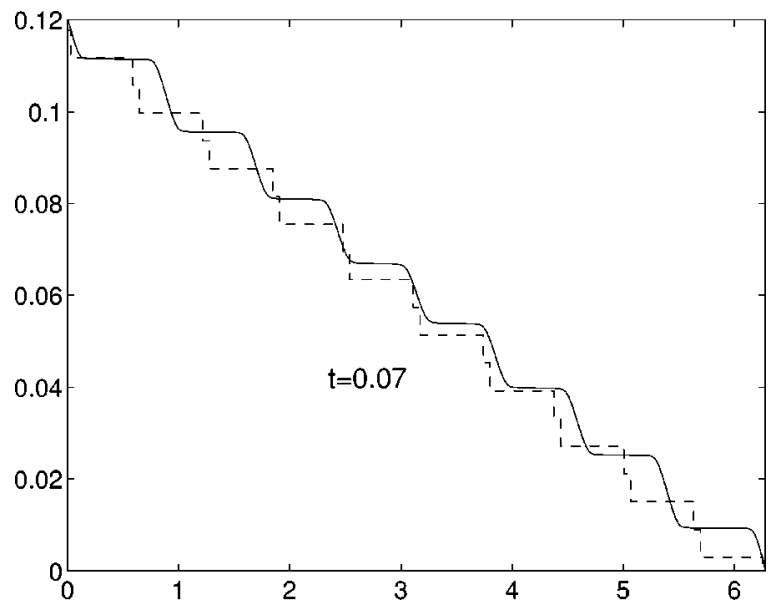

FIG. 5. Comparison of the results of our continuum model and the discrete model at time $t=0.07$ for $l_{e} / l=0.192$. The solid continuous surface is the result of our continuum model and the dashed stepped surface is the result of the discrete model. Here the length scale is that before rescaling, i.e., initially $h_{x}=-0.0192$.

continuum model agree with those of the discrete model qualitatively in the regime $l_{e} / l \ll 1$ and quantitatively in the regime $l_{e} / l \gg 1$.

These simulations are performed until $\min \left|h_{x}\right|$ becomes very small. The singularity in the continuum equation prevents us from simulating the further evolution of the step bunching. Physically, when the slope becomes very small (the terraces become very wide), nucleation will happen. In this case, we suggest that one can use the regularized misfit elastic energy (36) instead of Eq. (35). This will be left for the future work.

\section{EQUILIBRIUM SHAPES}

In this section, we compute the equilibrium step bunch shapes. As in the preceding section, we consider only the misfit and broken bond elastic interactions between steps.

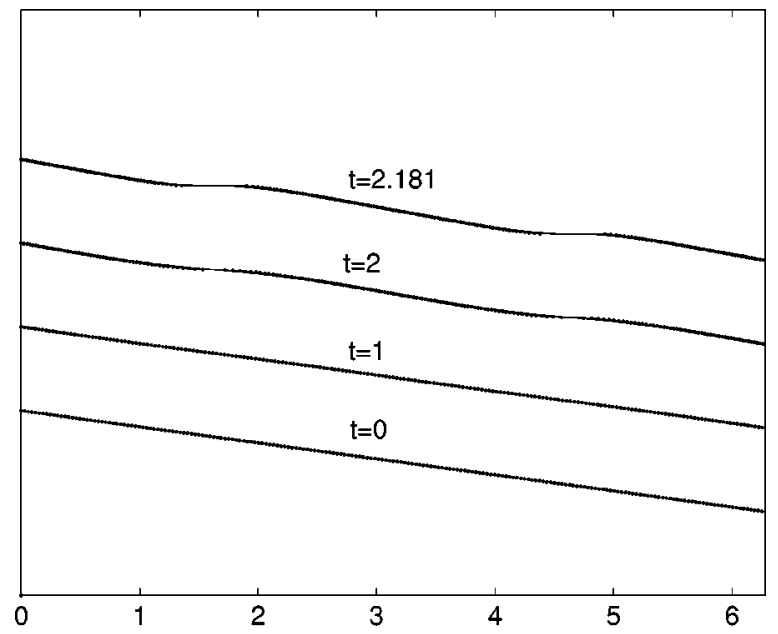

FIG. 6. Evolution of the surface when $l_{e} / l=2.732$. Solid line, result of our continuum model; dots, positions of steps from the discrete model. 

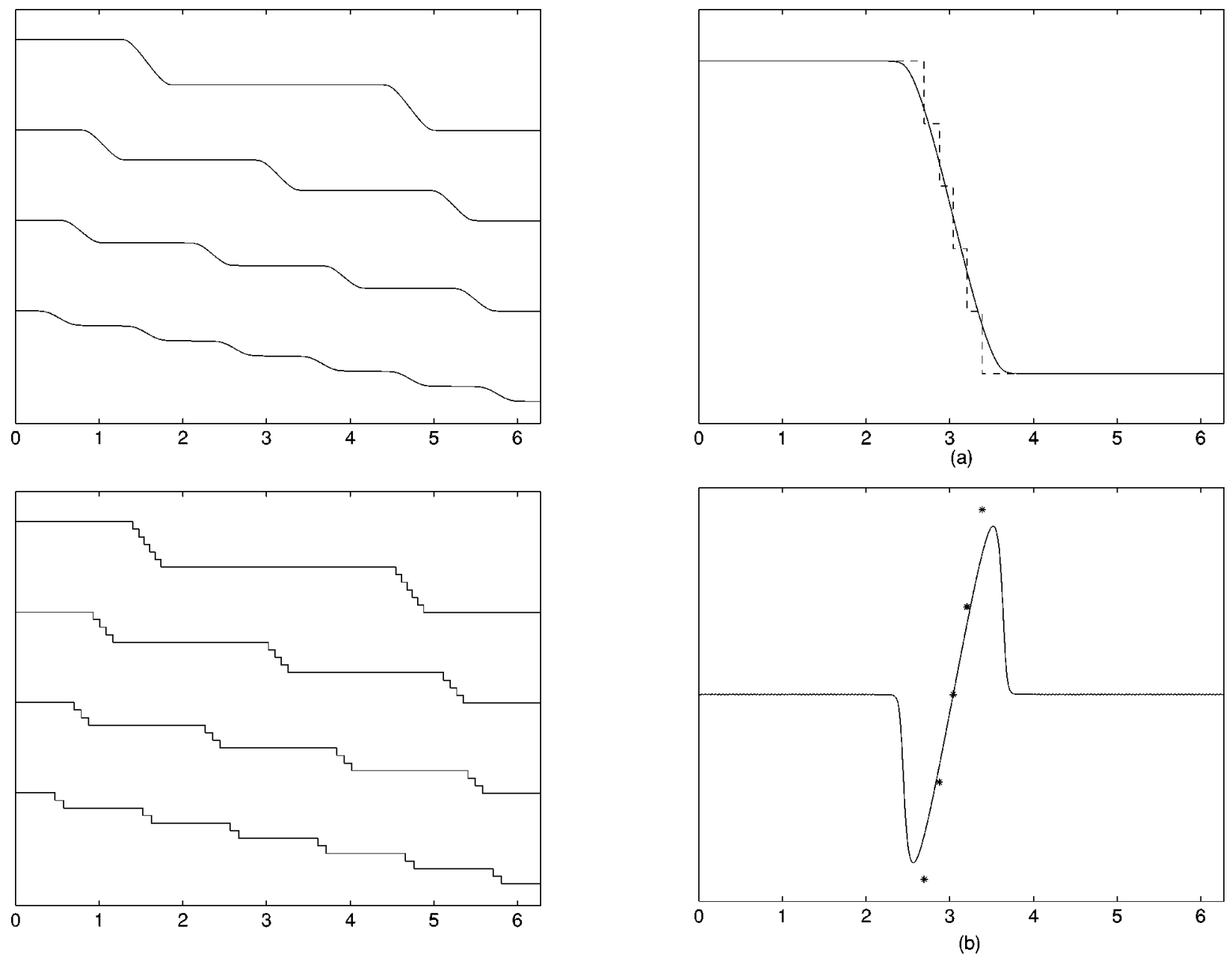

FIG. 7. Equilibrium shapes of step bunches computed using our continuum model and the discrete model for $r=0.365$ and $N$ $=2,3,4,6$.

The equation is Eq. (71) with two independent parameters $N$ and $r$. The initial planar surface is $h_{x}=-1$.

We can rewrite the Eq. (71) as

$$
h_{t}=\mu_{x x},
$$

where the chemical potential $\mu$ is

$$
\mu=-H\left(h_{x}\right)+\frac{1}{N}\left(\frac{1}{h_{x}}+r h_{x}\right) h_{x x} .
$$

It is the variation of the free energy

$$
E=\int\left(-\frac{1}{2} \tilde{h} H\left(h_{x}\right)+\frac{1}{N}\left|h_{x}\right| \ln \left|h_{x}\right|+\frac{1}{6} \frac{r}{N}\left|h_{x}\right|^{3}\right) d x
$$

where $\tilde{h}=h+x$ is the deviation from the initial planar surface.

The equilibrium step bunch shape satisfies the equation

$$
\mu=-H\left(h_{x}\right)+\frac{1}{N}\left(\frac{1}{h_{x}}+r h_{x}\right) h_{x x} \equiv \text { const. }
$$

FIG. 8. (a) Comparison of equilibrium step bunch shape for $r$ $=0.365$ and $N=5$. Solid line, result of our continuum model; dashed line, result of the discrete model. (b) Attractive force acting on the step bunch in (a). Solid line, result of our continuum model; stars, result of the discrete model.

We seek the periodic solutions to this equation using Newton's method. We also compute the equilibrium states using the discrete model of Tersoff et al. ${ }^{47}$ [Eq. (14) without deposition flux $F$ ] and compare the results obtained by these two models. More details of the numerical methods we use can be found in Xiang's thesis. ${ }^{62}$

In the first example, we fix $r=0.365\left(l=52 a, l_{e}=10 a\right)$, and vary $N$. These values of $l$ and $l_{e}$ were used by Tersoff et $a l .{ }^{47}$ The uniform step train is unstable from the linear instability result (critical value is $N=1.365$ ). The results are shown in Figs. 7-10. Figure 7 shows the equilibrium shapes of step bunches computed using our continuum model as well as the discrete model for $N=2,3,4,6$. Figure 8 shows the comparison of the equilibrium step bunch shape and attractive force acting on each step for $N=5$. They match very well. Figure 9 shows the average energy density for the uniform step train and for different sizes of step bunches. We can see that step bunching reduces the energy and larger step bunch has lower energy. This can be used to explain the formation of step bunches and why they prefer to coalesce. From Fig. 7 and Fig. 8(a) we see that a step bunch is almost 


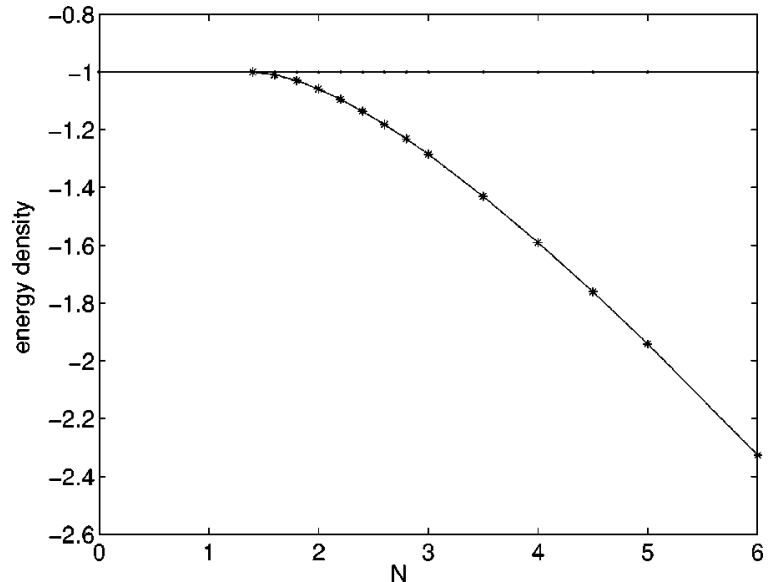

FIG. 9. Average energy density for the uniform step train (straight line) and for different sizes of step bunches (curved line) for $r=0.365$. The horizontal axis is the number of steps in a step bunch. The vertical axis is average energy density. We rescale the average energy density for the uniform step train to be -1 .

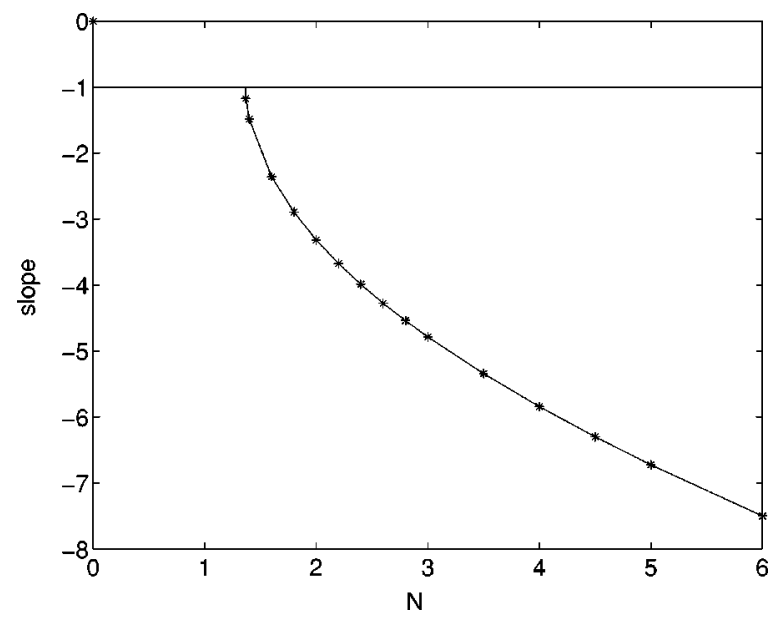

FIG. 10. Slopes of step bunches (curved line) and the slope of the uniform step train (straight line) vs $N$ for $r=0.365$.

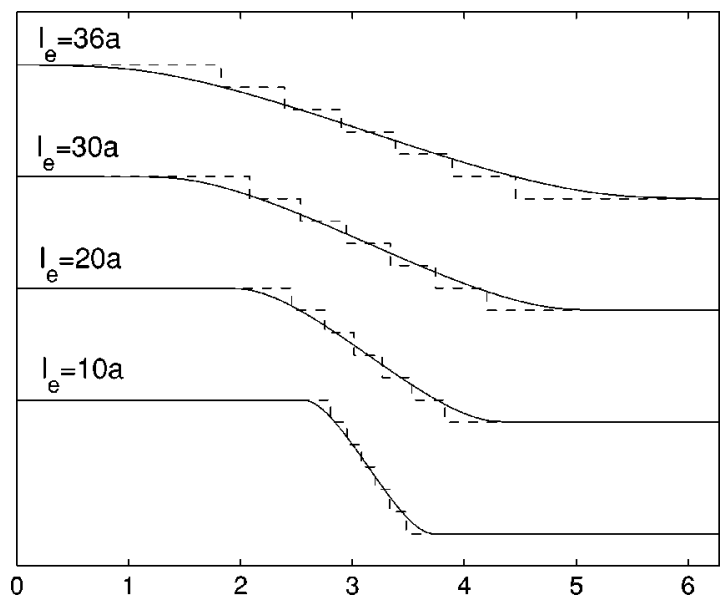

FIG. 11. Equilibrium step bunch shapes for $N=6, l=52 a$, and $l_{e}=10 a, 20 a, 30 a, 36 a$. Solid line, result of our continuum model; dashed line, result of the discrete model.

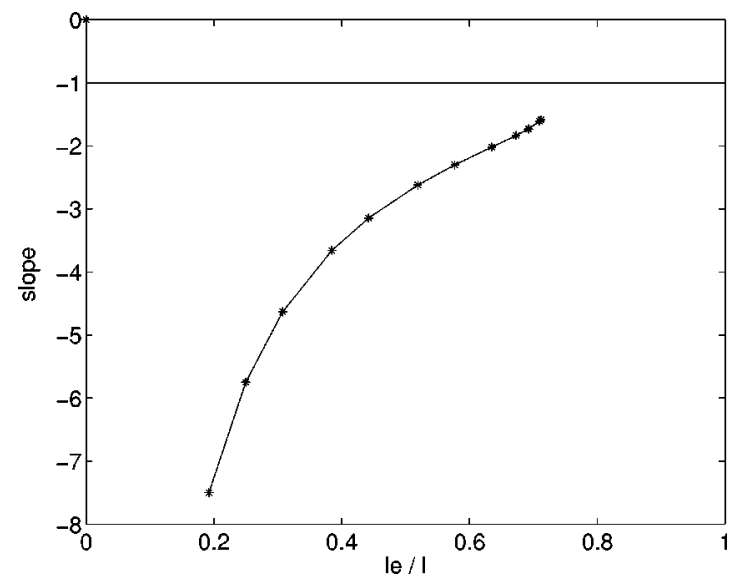

FIG. 12. Slopes of step bunches (curved line) and the slope of the uniform step train (straight line) vs $l_{e} / l$ for $N=6$.

straight, thus we can approximate its slope by the tangent slope in the middle and define this slope as the slope of this step bunch. Figure 10 shows the slopes of equilibrium step bunches varying with $N$ and the comparison with the slope of the initial uniform step train. We can see that a large step bunch is steeper than a smaller one. The critical value for $N$ is $N=1.365$, below which the uniform step train is stable.

In the second example, we fix $N=6$, and vary $r$ ( $l$ $=52 a$ fixed, $l_{e}$ varied). The results are shown in Figs. 11 and 12. We can see that the smaller the value of $l_{e}$, the steeper the step bunch. That is not a surprise because smaller value of $l_{e}$ means smaller repulsive interaction between steps so they can bunch more compactly. The results of our continuum model match very well with those of the discrete model. The critical value is $l_{e}=37.01 a(r=6)$, above which the planar surface is stable. ${ }^{65}$

In the third example, we compute the equilibrium step bunch shapes for $l_{e}>l\left(l=52 a, l_{e}=60 a, N=18\right)$. The result is shown in Fig. 13. Again the result of our continuum model matches very well with that of the discrete model.

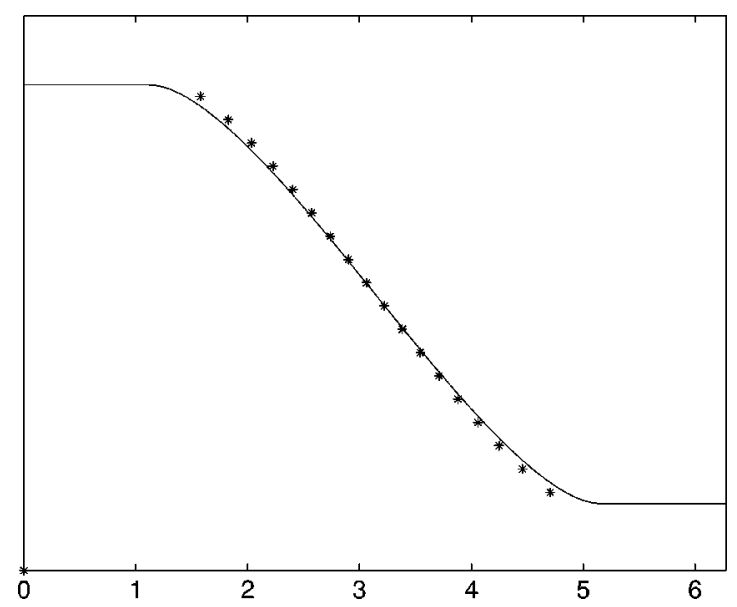

FIG. 13. Equilibrium step bunch shape for $N=18, l=52 a, l_{e}$ $=60 \mathrm{a}$. Solid line, result of our continuum model; stars, positions of steps obtained by the discrete model. 


\section{CONCLUSIONS}

In this paper, we have presented a continuum model governing step bunching induced by elasticity for $1+1$ dimensional monotonic vicinal surface. We have validated our model by performing linear stability analysis and numerical simulation in the nonlinear regime. We have also studied the equilibrium shapes of step bunches and explained their coalescence. We have presented a continuum description for the misfit elastic energy on vicinal surface which incorporates the underlying atomic features of the vicinal surface.

The result of linear instability analysis of our continuum model agrees with existing results of the discrete models of Duport et al. ${ }^{52,53}$ and Tersoff et al. ${ }^{47}$ It also recovers the well-known results of the traditional Asaro-Tiller-Grinfeld instability.

In comparison with the discrete model of Tersoff et al. ${ }^{47}$ where only the attractive and repulsive elastic interactions between steps are considered, our continuum model captures the long wavelength instability that produces step bunches, as well as the coalescence of these bunches as time evolves and nonlinear effects become important. In the regime where the equilibrium distance $l_{e}$ between two steps is less than the distance $l$ between two adjacent steps in the initial uniform step train, our results agree qualitatively with those of the discrete model, though the most unstable mode and the nonlinear evolution may not exactly agree due to the small number of steps in one period (relatively large lattice constant $a$ ). In the regime where $l_{e}$ is much larger than $l$, all the linear instability and nonlinear evolution results agree very well with those of the discrete model.

We have also studied the equilibrium shapes of step bunches and found that larger step bunch has lower energy; this explains why step bunches prefer to coalesce. This property is very similar to that of equilibrium islands caused by the traditional Asaro-Tiller-Grinfeld instability, which was found by Kukta and Freud ${ }^{26}$ and by Zhang and Bower. ${ }^{31}$ The equilibrium shape of a step bunch depends on two factors: the number of steps in it and the ratio $l_{e} / l$; larger step bunches and the step bunches with smaller ratio $l_{e} / l$ are steeper. We have also used the discrete model to compute the equilibrium shapes of step bunches and found that the results obtained by the two different methods agree very well.

\section{ACKNOWLEDGMENTS}

This work was supported by NSF and DARPA through the VIP program. We would like to thank Professor Robert V. Kohn of New York University for many helpful discussions.
*Electronic address: maxiang@ust.hk

${ }^{1}$ R.J. Asaro and W.A. Tiller, Metall. Trans. 3, 1789 (1972).

${ }^{2}$ M.A. Grinfeld, Dokl. Akad. Nauk. (SSSR) [Sov. Phys. Dokl. 31, 831 (1986)].

${ }^{3}$ M.A. Grinfeld, J. Nonlinear Sci. 3, 35 (1993).

${ }^{4}$ D.J. Srolovitz, Acta Metall. 37, 621 (1989).

${ }^{5}$ B.J. Spencer, P.W. Voorhees, and S.H. Davis, Phys. Rev. Lett. 67, 3696 (1991).

${ }^{6}$ B.J. Spencer, P.W. Voorhees, and S.H. Davis, J. Appl. Phys. 73, 4955 (1993).

${ }^{7}$ H. Gao, in Modern Theory of Anisotropic Elasticity and Applications, edited by J. Wu, T. Ting, and D. Barnett (SIAM, Philadelphia, 1991), p. 139.

${ }^{8}$ H. Gao, J. Mech. Phys. Solids 42, 741 (1994).

${ }^{9}$ P. Nozières, in Solids Far from Equilibrium, edited by C. Godreche (Cambridge University Press, Cambridge, 1992), p. 1.

${ }^{10}$ P. Nozières, J. Phys. I 3, 681 (1993).

${ }^{11}$ L.B. Freund and F. Jonsdottir, J. Mech. Phys. Solids 41, 1245 (1993).

${ }^{12}$ J. Grilhe, Acta Metall. Mater. 41, 909 (1993).

${ }^{13}$ B.I. Yakobson, J. Chem. Phys. 99, 6923 (1993).

${ }^{14}$ B. Caroli, C. Caroli, B. Roulet, and P.W. Voorhees, Acta Metall. 37, 257 (1989).

${ }^{15}$ P.H. Leo and R.F. Sekerka, Acta Metall. 37, 3119 (1989); 37, 3139 (1989).

${ }^{16}$ M. A. Grinfeld, Thermodynamic Methods in the Theory of Heterogeneous Systems (Longman, Sussex, 1991).

${ }^{17}$ M.A. Grinfeld, Europhys. Lett. 22, 723 (1993).

${ }^{18}$ M.A. Grinfeld, Phys. Rev. B 49, 8310 (1994).

${ }^{19}$ W.H. Yang and D.J. Srolovitz, Phys. Rev. Lett. 71, 1593 (1993).

${ }^{20}$ W.H. Yang and D.J. Srolovitz, J. Mech. Phys. Solids 42, 1551 (1994).
${ }^{21}$ C.H. Chiu and H. Gao, Int. J. Solids Struct. 30, 2983 (1993).

${ }^{22}$ B.J. Spencer and D.I. Meiron, Acta Metall. Mater. 42, 3629 (1994).

${ }^{23}$ K. Kassner and C. Misbah, Europhys. Lett. 28, 245 (1994).

${ }^{24}$ B.J. Spencer and J. Tersoff, in Evolution of Epitaxial Structure and Morphology, edited by A. Zangwill, D. Jesson, D. Chambliss, and R. Clarke, Mater. Res. Soc. Symp. Proc. 339 (Materials Research Society, Pittsburgh, 1996), p. 283.

${ }^{25}$ B.J. Spencer and J. Tersoff, Phys. Rev. Lett. 79, 4858 (1997).

${ }^{26}$ R.V. Kukta and L.B. Freund, J. Mech. Phys. Solids 45, 1835 (1997).

${ }^{27}$ B.J. Spencer, Phys. Rev. B 59, 2011 (1999).

${ }^{28}$ C.D. Rudin and B.J. Spencer, J. Appl. Phys. 86, 5530 (1999).

${ }^{29}$ L.L. Shanahan and B.J. Spencer, Interfaces Free Boundaries 4, 1 (2002).

${ }^{30}$ C.H. Chiu and H. Gao, in Thin Films: Stresses and Mechanical Properties V, edited by S.P. Baker, P. Børgesen, P.H. Townsend, C.A. Ross, and C.A. Volkert, Mater. Res. Soc. Symp. Proc. 356 (Materials Research Society, Pittsburgh, 1995), p. 33.

${ }^{31}$ Y.W. Zhang and A.F. Bower, J. Mech. Phys. Solids 47, 2273 (1999).

${ }^{32}$ A. Pimpinelli and J. Villain, Physics of Crystal Growth (Cambridge University Press, New York, 1998).

${ }^{33}$ J. Tersoff and F.K. LeGoues, Phys. Rev. Lett. 72, 3570 (1994).

${ }^{34}$ Y.H. Xie, G.H. Gilmer, C. Roland, P.J. Silverman, S.K. Buratto, J.Y. Cheng, E.A. Fitzgerald, A.R. Kortan, S. Schuppler, M.A. Marcus, and P.H. Citrin, Phys. Rev. Lett. 73, 3006 (1994).

${ }^{35}$ T. Marschner, S. Lutgen, M. Volk, W. Stolz, E.O. Gobel, N.Y. Jin-Phillipp, and F. Phillipp, Superlattices Microstruct. 15, 183 (1994).

${ }^{36}$ Y.H. Phang, C. Teichert, M.G. Lagally, L.J. Peticolas, J.C. Bean, and E. Kasper, Phys. Rev. B 50, 14435 (1994). 
${ }^{37}$ K. Hiramoto, T. Tsuchiya, M. Sagawa, and K. Uomi, J. Cryst. Growth 145, 133 (1994).

${ }^{38}$ T. Kikkawa, K. Makiyama, H. Ochimizu, K. Kasai, and J. Komeno, J. Cryst. Growth 145, 799 (1994).

${ }^{39}$ G.S. Bales and A. Zangwill, Phys. Rev. B 41, 5500 (1990).

${ }^{40}$ R.L. Schwoebel, J. Appl. Phys. 40, 614 (1969).

${ }^{41}$ M.S. Miller, H. Weman, C.E. Pryor, M. Krishnamurthy, P.M. Petroff, H. Kroemer, and J.L. Merz, Phys. Rev. Lett. 68, 3464 (1992)

${ }^{42}$ V. Bressler-Hill, A. Lorke, S. Varma, P.M. Petroff, K. Pond, and W.H. Weinberg, Phys. Rev. B 50, 8479 (1994).

${ }^{43}$ K. Pond, A.C. Gossard, A. Lorke, and P.M. Petroff, Mater. Sci. Eng., B 30, 121 (1995).

${ }^{44}$ T. Jung, R. Schlittler, J.K. Gimzewski, and F.J. Himpsel, Appl. Phys. A: Mater. Sci. Process. A61, 467 (1995).

${ }^{45}$ C. Teichert, M.G. Lagally, L.J. Peticolas, J.C. Bean, and J. Tersoff, Phys. Rev. B 53, 16334 (1996).

${ }^{46}$ C. Teichert, J.C. Bean, and M.G. Lagally, Appl. Phys. A: Mater. Sci. Process. A67, 675 (1998).

${ }^{47}$ J. Tersoff, Y.H. Phang, Z. Zhang, and M.G. Lagally, Phys. Rev. Lett. 75, 2730 (1995).

${ }^{48}$ F. Liu, J. Tersoff, and M.G. Lagally, Phys. Rev. Lett. 80, 1268 (1998).

${ }^{49}$ W.K. Burton, N. Cabrera, and F. Frank, Phil. Trans. Roy. Soc. 243, 299 (1951).

${ }^{50}$ J. Tersoff, Phys. Rev. Lett. 80, 2018 (1998).

${ }^{51}$ C. Duport, Ph.D. thesis, Université Joseph Fourier, Grenoble, France, 1996.

${ }^{52}$ C. Duport, P. Nozières, and J. Villain, Phys. Rev. Lett. 74, 134 (1995).
${ }^{53}$ C. Duport, P. Politi, and J. Villain, J. Phys. I 5, 1317 (1995).

${ }^{54}$ V.M. Kaganer and K.H. Ploog, Solid State Commun. 117, 337 (2001).

${ }^{55}$ V.M. Kaganer and K.H. Ploog, Phys. Rev. B 64, 205301 (2001).

${ }^{56}$ C. Duport, C. Priester, and J. Villain, in Morphological Organization in Epitaxial Growth and Removal, edited by Z. Zhang and M. Lagally (World Scientific, Singapore, 1997), p. 73.

${ }^{57}$ R.V. Kukta and K. Bhattacharya, Thin Solid Films 357, 35 (1999).

${ }^{58}$ W.W. Mullins, J. Appl. Phys. 28, 333 (1957).

${ }^{59}$ V. Holy, A.A. Darhuber, J. Stangl, G. Bauer, J. Nutzel, and G. Abstreiter, Phys. Rev. B 57, 12435 (1998).

${ }^{60}$ V.B. Shenoy and L.B. Freund, J. Mech. Phys. Solids 50, 1817 (2002).

${ }^{61}$ Y. Xiang, SIAM J. Appl. Math. 63, 241 (2002).

${ }^{62}$ Y. Xiang, Ph.D. thesis, New York University, New York, 2001.

${ }^{63}$ We keep one more higher term of $a$ in the misfit interaction between steps, which turns out to have the same order as the broken bond interaction.

${ }^{64}$ Figure 3 shows the instability in a different way from Fig. 2. In Fig. 3, the horizontal axis is $N$ (proportional to wavelength), while in Fig. 2, the horizontal axis is wave number $k$.

${ }^{65}$ Below the critical value of $l_{e}$, the energy variation of the small perturbation from the planar surface $\Delta E<0$, the planar surface is unstable. In the critical case $\Delta E \approx 0$. After checking the higher-order terms of $\Delta E$ for the critical case, we find $\Delta E<0$. Therefore in this case we can still have an equilibrium state other than the planar surface. That is why there is a jump of slope at the critical value in Fig. 12. 